\section{Take a breath}

Is it time to re-think how we diagnose COPD?

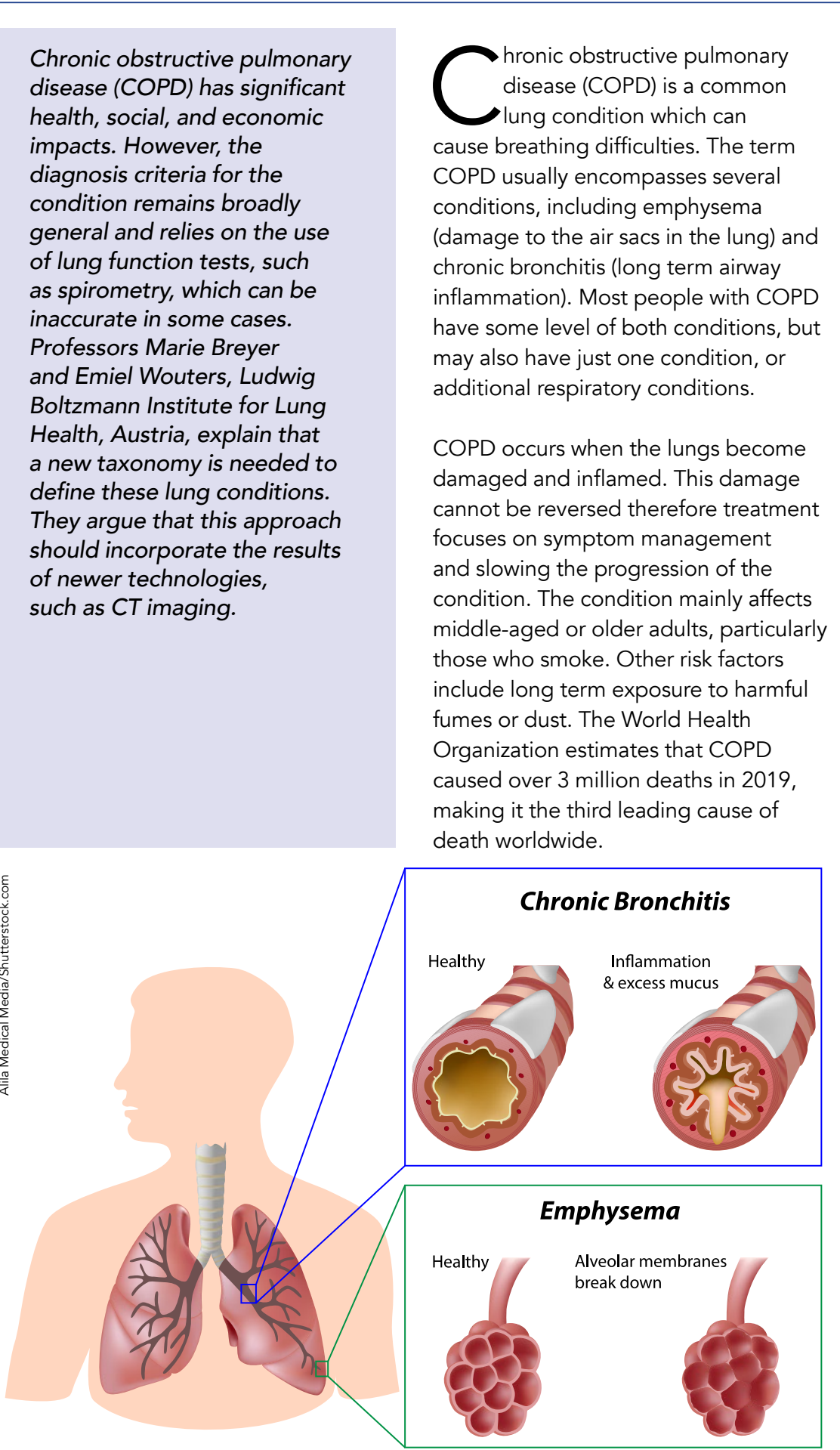

Symptoms include breathlessness, persistent cough, frequent chest infections, and wheezing. There may be periods when these symptoms are worse, for example during an infectious

\section{SPIROMETRY}

One way that COPD is diagnosed is Spirometry measures lung function, including the volume and flow (speed) of air that can be inhaled and exhaled. The patient takes a deep breath and exhales into the sensor as hard, and for as long as they can. The technique generates charts that show the volume and flow of air during one inhalation and one exhalation. These results can be used to measure breathing pattern over time, or to identify breathing patterns that correlate with lung conditions such as asthma, pulmonary

\section{PRE-COPD}

The FEV1/FVC ratio reflects the amoun of air that you can forcefully exhale for your lungs (the ratio of the forced the for in one second (FEV) lungs). The forced vital capacity is the total volume of air exhaled during the test, and this value is compared with the volume expired in the first second of the test.

has been suggested that some individuals may have 'pre-COPD'. This is when there is no aifflow obstruction identified with spirometry.

However, Professors Breyer and Wouters at the Ludwig Boltzmann the labelling of a new condition. A new clinical category of COPD (pre-
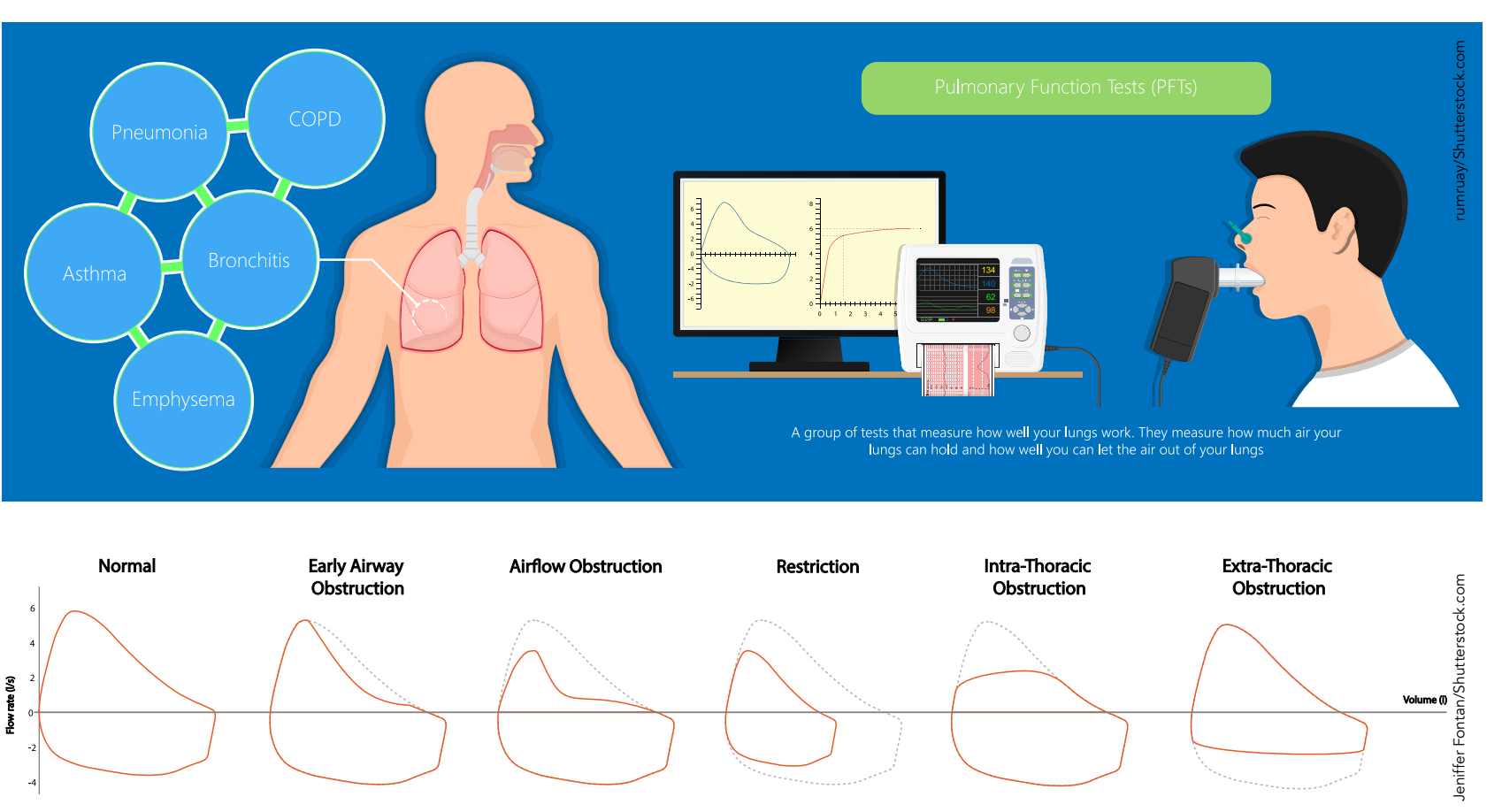

The definitions of COPD and COPD-related
account that particular individual's status.

COPD) would require a clear definition

as well as defined cut-off values for

measurements of any tests used to

diagnose the condition. Instead, they

suggest that a new taxonomy may.

be needed for all chronic respiratory conditions, as opposed to the possible, and

proach, taking in introduction of new pre-diseases.

from this diagnosis. They may not get the has progressed to a stage where it is impacting airflow.

has on mortality rates, the definition of Using physiological definitions of $\begin{array}{ll}\text { the condition is largely descriptive. It } & \begin{array}{l}\text { disease can lead to inaccuracy and } \\ \text { states that aifflow obstruction (the inability } \\ \text { insensitivity of diagnoses. The Ludwig }\end{array}\end{array}$

While many

conditions

are currently

diagnosed and

characterised

by spirometry

emphasises the

Despite the significant impact that COPD has on mortality rates, the definition of the condition is largely descriptive.

nstitute for

need to integrate new physiological- and

One example of a new technology is computed tomography (CT scanning), which is often used to detect emphysema. However, Breyer and Wouters explain tha clear definitions are needed to delineate between sub-types of emphysema.

There are opportunities to integrate more sensitive diagnostic procedures into the COPD diagnostic pathway. This may allow a variety of abnormalities to be identied early in the disease process. Identifying and characterising that treanges in ling function mean

to move air in and out of the lungs effectively) is a major indicator of COPD. Professors Breyer and Wouters explain early-stage disease and who are not yet showing aifflow obstruction, are excluded

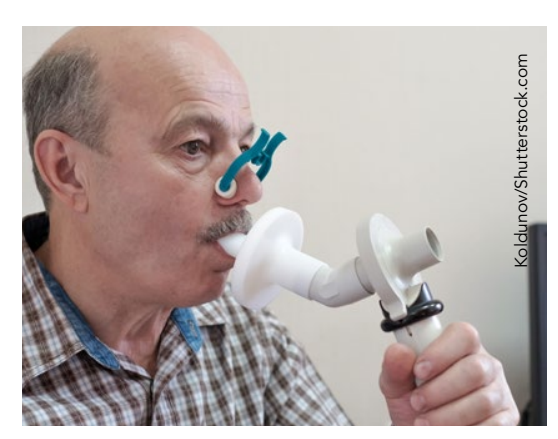

Spirometry assesses how well your lun
exhale and how $h$ w wichly you exhate
Lung Health researcher

emphasise that the

labelling of disease

is a fluid process,

which may change

in response to new discoveries. However,

It is important to be able to accurately define and diagnose a condition, as here are significant emotional, social, nd financial implications to diagnosing someone (correctly or incorrectly) with a

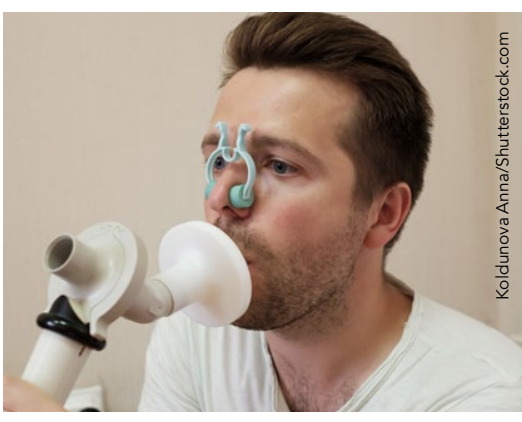


